4. G. D. Mostow, On the fundamental group of a homogeneous space, Ann. of Math. (2) vol. 66 (1957) pp. 249-255.

5. E. Specker, Die erste Cohomologie gruppe von Uberlagerungen und Homotopieeigenschaften dreidimensionaler Mannigfaltigkeiten, Comment. Math. Helv. vol. 23 (1949) pp. 303-333.

6. P. A. Smith, The fundamental group of a group manifold, Ann. of Math. (2) vol. 36 (1935) pp. 210-229.

7. - Manifolds with abelian fundamental groups, Ann. of Math. (2) vol. 37 (1936) pp. 526-533.

UNIVERSITY OF Michigan

\title{
ON THE GROUP OF AFFINITIES OF LOCALLY AFFINE SPACES
}

\section{LOUIS AUSLANDER ${ }^{1}$}

Let $M$ be a compact manifold with a given complete flat affine connection (i.e., an affine connection with curvature and torsion zero). Then we may represent the fundamental group $\Gamma$ of $M$ by affine transformations of the real affine space $R^{n}$, in such a way that the orbit space of $R^{n}$ by $\Gamma$ is homeomorphic to $M$. We will denote the full group of affine transformations of $R^{n}$ by $A(n)$ and the orbit space of $R^{n}$ under $\Gamma$ by $R^{n} / \Gamma$. We represent the elements of $A(n)$ as matrices of the form

$$
\left(\frac{A}{0} \mid \frac{v}{1}\right)
$$

where $A$ is the (nonsingular) linear transformation part and $v$ is the translational part. Let $G$ be the group of all affinities of $M$, i.e., the group of all homeomorphisms of $M$ onto itself which preserve the given affine structure on $M$. Nomizu proved in [3] that $G$ is a Lie group. Let $G_{1}$ denote the identity component of $G$. It is the purpose of this note to prove that $G_{1}$ is a nilpotent Lie group.

Now it is well known that any map of $M$ into itself can be lifted to a map of $R^{n}$ into itself, uniquely up to covering transformations, i.e., up to elements of $\Gamma$. The maps in $G_{1}$ lift to affine transformations of $R^{n}$. It is clear that $G^{*}$, the identity component of the subgroup of $A(n)$ so obtained, projects back onto $G_{1}$ as a covering group. Further, since $g^{*} \Gamma g^{*-1}=\Gamma$, for all $g^{*} \in G^{*}$ and since $G^{*}$ is connected and $\Gamma$ discrete, it follows easily that $G^{*}$ and $\Gamma$ commute elementwise.

Received by the Editors January 4, 1957.

1 This paper was written while the author was a National Science Foundation Post Doctoral Fellow. 
Lemma 1. There exist $\gamma_{i} \in \Gamma, i=1, \cdots, n$, whose translational components are linearly independent.

Proof. Assume the lemma is false and all translational components lie in a linear subspace $V \subset R^{n}$. Then $V$ must be invariant by $\Gamma$ that is, we have $\gamma(V) \subset V$ for all $\gamma \in \Gamma$. In $R^{n}$ choose a compact fundamental domain $D$ for $R^{n} / \Gamma$. Then $V \cap D$ is compact and a fundamental domain for $\Gamma$ restricted to $V$. Hence $V / \Gamma$ must be a compact manifold of dimension less than $n$ with fundamental group $\Gamma$. Using the theorem of Eilenberg-MacLane on groups operating on acyclic spaces [2], we see that the $n$ dimensional cohomology group of the group $\Gamma$ with coefficients integers modulo 2, must be zero. But this contradicts the fact that $\Gamma$ is also the fundamental group of an $n$ dimensional manifold with $R^{n}$ as universal covering space.

Lemma 2. Let $g^{*} \in G^{*}$ be such that $g^{*}\left(x_{0}\right)=x_{0}$ for some $x_{0} \in R^{n}$. Then $g^{*}$ is the identity element of $G^{*}$.

Proof. Let $g^{*}\left(x_{0}\right)=x_{0}$. Choose $x_{0}$ as the origin of the coordinate system. Now $g^{*} \gamma\left(x_{0}\right)=\gamma g^{*}\left(x_{0}\right)=\gamma\left(x_{0}\right)$. Hence $g^{*}$ leaves the images of $x_{0}$ under $\Gamma$ point-wise fixed. Since the points $\gamma\left(x_{0}\right), \gamma \in \Gamma$ span $R_{n}$ by Lemma $1, g^{*}$ is the identity element of $G^{*}$.

Theoreu. Let $M$ be a complete compact flat affine space. Let $G$ be its group of affinities. Then the identity component of $G$ is a nilpotent Lie group.

This is equivalent to proving that $G^{*}$ is a nilpotent Lie group. Now $G^{*} \subset A(n)$. Let $C^{n}$ denote the $n$-dimensional affine space over the complex field; the corresponding group of affine transformations will be denoted by $A(n, C)$. Then $A(n)$ may be considered as a subgroup of $A(n, C)$. Since $g^{*} \in G^{*}$ operates without fixed points in $R^{n}$, it will do so also in $C^{n}$. Further $\gamma g^{*}=g^{*} \gamma$.

Let $\exp (M t)$ be a one parameter subgroup of $G^{*}$ with infinitesimal generator $M$. The matrix $M$ can be assumed in normal form

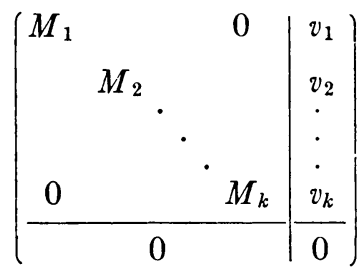

where each $M_{i}$ is triangular with all eigenvalues equal.

Lemma 3. All eigenvalues of $M$ are 0 ; i.e., $M$ is nilpotent. 
Proof. By changing the origin, if necessary, we can assume that the translational part $v_{i}$ is 0 for every $M_{i}$ with eigenvalue different from 0 . At least one eigenvalue must be 0 , otherwise the elements $\exp (M t)$ would have the origin as fixed point; this would contradict Lemma 2. Because $M$ has the above special form, it is easy to see that the coordinates of the points on the orbit of the origin under $\exp (M t)$ are given by polynomials in $t$. Suppose that there is an $M_{i}$ with eigenvalue not zero. Let $y$ be any point with a nonzero coordinate corresponding to the element in position $(1,1)$ of $M_{i}$. But then on the orbit of $y$ under $\exp (M t)$ this coordinate has the form $C \cdot \exp \left(\lambda_{i} t\right)$, with $C \neq 0$. Applying Lemma 1 , one sees that there exists a $\gamma \in \Gamma$ such that for the orbit of $\gamma(0)$ at least one coordinate is of exponential form. But because $g^{*}(\gamma(0))=\gamma\left(g^{*}(0)\right)$ and the remark on the orbit of 0 above, the coordinates on the orbit of $\gamma(0)$ must be polynomials. This proves Lemma 3.

Our theorem is now an immediate consequence of the well-known fact that a linear Lie algebra, all of whose elements are nilpotent, is nilpotent [4].

\section{BIBLIOGRAPHY}

1. L. Auslander and I. Singer, Homogeneous spaces with invariant flat affine connections (in preparation).

2. S. Eilenberg and S. MacLane, Relations between homology and homotopy groups of spaces, Ann. of Math. vol. 46 (1945) pp. 480-509.

3. K. Nomizu, On the group of affine transformations of an affinely connected manifold, Proc. Amer. Math. Soc. vol. 4 (1953) pp. 816-823.

4. Seminaire, Sophus Lie, Ecole Normale Superieure, 1954-1955.

The Institute for Advanced Study and INDIANA UNIVERSITY 